\title{
NONLINEAR ELECTRON SOLUTIONS AND THEIR CHARACTERISTICS AT INFINITY
}

\author{
HILARY BOOTH ${ }^{1}$
}

(Received 1 May, 2000; revised 7 August, 2000)

\begin{abstract}
The Maxwell-Dirac equations model an electron in an electromagnetic field. The two equations are coupled via the Dirac current which acts as a source in the Maxwell equation, resulting in a nonlinear system of partial differential equations (PDE's). Well-behaved solutions, within reasonable Sobolev spaces, have been shown to exist globally as recently as 1997 [12]. Exact solutions have not been found-except in some simple cases.

We have shown analytically in $[6,18]$ that any spherical solution surrounds a Coulomb field and any cylindrical solution surrounds a central charged wire; and in [3] and [19] that in any stationary case, the surrounding electron field must be equal and opposite to the central (external) field. Here we extend the numerical solutions in [6] to a family of orbits all of which are well-behaved numerical solutions satisfying the analytic results in [6] and [11]. These solutions die off exponentially with increasing distance from the central axis of symmetry. The results in [18] can be extended in the same way. A third case is included, with dependence on $z$ only yielding a related fourth-order ordinary differential equation (ODE) [3].
\end{abstract}

\section{The Maxwell-Dirac equations}

The Maxwell-Dirac equations model an electron in an electromagnetic field. The two equations are coupled via the Dirac current $j_{\alpha}$, that is, we include the nonlinearity of the self-field, (see (1)). Global existence was fully established by Flato, Simon and Taflin as recently as 1997 [.12], despite sustained interest $([7,13-15])$ in this nonlinear system of PDE's.

In covariant notation (using the summation convention), the coupled Maxwell-Dirac equations can be written as follows:

$$
\begin{gathered}
\gamma^{\alpha}\left(\partial_{\alpha}-i e A_{\alpha}\right) \psi+i m \psi=0, \quad \alpha=0, \ldots, 3, \\
F_{\alpha \beta}=A_{\beta, \alpha}-A_{\alpha, \beta}, \quad \partial^{\alpha} F_{\alpha \beta}=-4 \pi e j_{\beta}, \quad \text { where } j_{\alpha}=\bar{\psi} \gamma_{\alpha} \psi .
\end{gathered}
$$

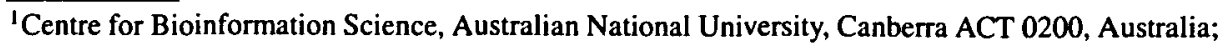
e-mail: hilary.booth@wintermute.anu.edu.au.

(C) Australian Mathematical Society 2002, Serial-fee code 1446-1811/02 
Note that $\psi \in C^{4}$ is the Dirac wave-function or 4-spinor and $\bar{\psi}$ the Dirac conjugate. These are acted upon by $\gamma^{\alpha}$ which are the usual gamma matrices (members of a Clifford algebra) and $A_{\alpha}$ is the 4-potential.

Quantum electrodynamics (QED) are based upon these equations. In QED, the interactions are introduced as a perturbation expansion and evaluated using Feynman integrals (or diagrams). However, without second quantization of the field, various authors have sustained interest in the answers to several questions:

(1) Do well-behaved solutions exist ([7,12-15])?

(2) What do these solutions look like, at the origin and at infinity $([6,18])$ ?

(3) Do these characteristics have any physical meaning $([6,18])$ ?

(4) Can we find numerical solutions $([6,16,18,21])$ ?

1.1. Some simplified versions of the problem The full four-dimensional nonlinear problem is somewhat intractable-global existence has only been established recently ([12] and references therein). Some simplified versions of the problem are as follows:

(1) The static case in which we assume that there exists a Lorentz frame in which there is no current "flow" $([6,18])$, that is, $j^{\alpha}=\delta_{0}^{\alpha} j^{0}$.

(2) The stationary case [11] in which we assume $\psi\left(x_{0}, x\right)=e^{i \omega x_{0}} \phi(x)$.

(3) The static spherically symmetric case ([18]).

(4) The static cylindrically symmetric case ([6]).

In keeping with the theorem in [19] which outlines the conditions under which the static condition implies stationarity, cases (3) and (4) are also stationary. Other twodimensional versions are the $1+1$ case which was solved exactly for a massless electron by Schwinger in [20], the axi-symmetric case and a slightly more general version in which we assume circular current flow $j^{\alpha}=\left(j^{0}, 0, j^{\phi}, 0\right)$, all of which require further investigation.

\section{ODE solutions}

Even within the axi-symmetric system there are three interesting ODE cases, spherically symmetric, cylindrically symmetric and dependence on $z$ only. The spherical and cylindrical cases were examined extensively in $[6,18]$. The case where dependence is on $z$ only, is similar in some respects ([3]). We first apply reality conditions to the electromagnetic potential $A^{\alpha}$ which has been expressed in terms of Dirac spinors and their first derivatives (by solving the Dirac equations for the potential $([18,19])$ ). These reality conditions allow us some simpler expressions which are then inserted into the Maxwell equation, resulting in fourth-order ODE's. We will also note here that in the $1+1$ case $([4,9,10])$ the system was also reduced to fourth-order ODE's, which 
in some cases are solved explicitly $([9,10])$, whilst in other cases we are currently developing more numerical results ([4]).

When we assume that the Dirac current is static, we lose three (real) degrees of freedom in $\psi$, since three components of $j^{\alpha}$ are set to zero. See [6] and [18] in which we also fix the gauge by choosing

$$
\begin{array}{ll}
\psi^{0}=-Y e^{i(\chi-\eta) / 2}, & \psi^{2}=-Y e^{-i(x+\eta) / 2}, \\
\psi^{1}=X e^{i(x+\eta) / 2}, & \psi^{3}=X e^{-i(x-\eta) / 2},
\end{array}
$$

where $\eta, \chi, X$ and $Y$ are real functions. These expressions were substituted into the potential (which was solved for in terms of Dirac spinors and their first derivatives), yielding

$$
\begin{aligned}
A^{0} & =\cos \chi+\frac{\left(X^{2}-Y^{2}\right)}{\left(X^{2}+Y^{2}\right)} \frac{\partial \eta}{\partial t}+\frac{(\nabla \chi) \cdot l}{\left(X^{2}+Y^{2}\right)} \\
A & =\frac{1}{\left(X^{2}+Y^{2}\right)}\left[\frac{\partial \chi}{\partial t} l+\left(X^{2}-Y^{2}\right) \nabla \eta-\nabla \times l\right] .
\end{aligned}
$$

We can now demand that the null tetrads $l^{\alpha}, n^{\alpha}, m^{\alpha}$ (and $\bar{m}^{\alpha}$ ) are real. This results in the three reality conditions following. These conditions are written in general form in [18]. Note that these reality conditions can also be regarded as three of four consistency conditions resulting from having eight equations (the Dirac equations and their four conjugate equations) in four unknowns (the four terms of $A^{\alpha}$ ), so that when we solve for the potential we must demand that these conditions are met for consistency. In [5] we look at these conditions and show their equivalence to Radford's reality conditions, before generalizing them to higher dimensions. One of the conditions is conservation of charge, which is obeyed automatically as stated in [18]. The other three conditions, in the variables required for the static case, are given below:

$$
\begin{gathered}
\frac{\partial}{\partial t}\left(X^{2}+Y^{2}\right)=0, \\
\nabla . l=-\left(X^{2}+Y^{2}\right) \sin \chi, \\
\frac{\partial l}{\partial t}+(\nabla X) \times l=0,
\end{gathered}
$$

where $l=\left(2 X Y \cos \eta, 2 X Y \sin \eta, X^{2}-Y^{2}\right)$.

The Maxwell equations act upon $A$, as defined above, and the current vector becomes $j^{\alpha}=\left(2\left(X^{2}+Y^{2}\right), 0,0,0\right)$.

We showed in [3] that the static equations, in the gauge given by (2), are stationary if and only if $\partial \eta / \partial t=0$ and $\partial X / \partial t=0$ (or $\partial Y / \partial t=0$ ). In the stationary case, $\partial \chi / \partial t=0$ and $\partial l / \partial t=0$. Now in the stationary case, the third reality condition (7) tells us that $\nabla_{\chi}$ is proportional to $l$ and we choose the function $\alpha$ such that 
$l=(\alpha / r \sin \theta) \nabla \chi$. Substituting this into the expression for the potential (3) and noting that $X^{2}+Y^{2}=|l|$ then

$$
A^{0}=\cos \chi \pm \frac{\nabla \chi \cdot l}{\left(X^{2}+Y^{2}\right)}=\cos \chi \pm \sqrt{\chi_{r}^{2}+\frac{\chi_{\theta}^{2}}{r^{2}}+\frac{\chi_{\phi}^{2}}{r^{2} \sin ^{2} \theta}} .
$$

From here, it is a straightforward calculation to apply symmetry arguments and calculate the resulting ODE's $([3,6,18])$. All three cases can be expressed in the following form, where

$$
x= \begin{cases}r, \text { distance from origin, } & \text { in the spherical case; } \\ \rho, \text { distance from central axis, } & \text { in the cylindrical case; } \\ z, & \text { in the } z \text { dependent case. }\end{cases}
$$

Then, in dimensionless variables $([6,18])$, the equations reduce to:

$$
\frac{d \chi}{d x}=A-\cos \chi, \quad \frac{d F}{d x}=Z, \quad \frac{d A}{d x}=\frac{F}{f(x)}, \quad \frac{d Z}{d x}=-Z \sin \chi,
$$

where

$$
f(x)= \begin{cases}x^{2}, & \text { in the spherical case; } \\ x, & \text { in the cylindrical case } \\ 1, & \text { in the } z \text { dependent case. }\end{cases}
$$

It is easily shown that these four first-order equations can be written as the fourth-order equation,

$$
\frac{d^{2}}{d x^{2}}\left(f(x)\left(\frac{d^{2} \chi}{d x^{2}}-\sin \chi \frac{d \chi}{d x}\right)\right)+\frac{d}{d x}\left(f(x)\left(\frac{d^{2} \chi}{d x^{2}}-\sin \chi \frac{d \chi}{d x}\right)\right) \sin \chi=0 .
$$

In the cylindrical case, $Z(\rho)$ is the charge per unit ring radius $\rho, F(\rho)$ is the charge within a radius $\rho$, and $A$ is the scalar potential, $A^{0}$ (see [6]). Similar physical quantities are represented in the spherical and $z$ cases. Most importantly, we are looking for solutions whose charge density $Z(x)$ decreases rapidly towards infinity, so that we can find solutions which are localized or particle-like. Our zero total charge result [3] tells us to expect that $F \rightarrow 0$ as $x \rightarrow \infty$ and likewise, $A \rightarrow$ const as $x \rightarrow \infty$.

As pointed out by Chris Cosgrove (private communication), (10) has non-integer resonance numbers $([1,2])$ and we do not expect to find an integrable system (in the soliton sense) here. Instead, we show that there are a family of orbits (in the sense of [8]) all of which approach the trivial (constant) solution at infinity. As an example, we look at the cylindrical case, noting that similar results hold in the spherical and $z$ case. The orbits in the following section complete the results in [6] in which a single member (analytic in $1 / \rho$ ) of these families was shown to exist and calculated numerically. 


\section{Numerical solutions (cylindrical case)}

In [6] the following results were shown.

LEMMA 1. Suppose $(\chi, F, A, Z)$ is a solution to (9) on $I=\left(0, \rho_{1}\right)$, for some $\rho_{1}$, $0<\rho_{1}<1$. Suppose also that $Z \geq 0$ is continuous and bounded on $I$. Then

(i) $F$ is $C^{1}$ on I and has a well-defined, finite limit as $\rho \rightarrow 0$. Z has a well-defined limit as $\rho \rightarrow 0$.

(ii) If $F(0) \neq 0$ then $A$ is unbounded as $\rho \rightarrow 0$. In particular, $A=\Omega(\rho) \ln (\rho)$, where $\Omega$ is $C^{2}$ and bounded on $I, \Omega \rightarrow F(0)$ as $\rho \rightarrow 0$. Also, $\chi$ is bounded as $\rho \rightarrow 0$.

LEMMA 2. Suppose $(\chi, F, A, Z)$ is a solution to (9) on $\rho \in(0, \infty)$. Suppose also that $Z \geq 0$ with $F$ continuous and bounded on the interval. Then

(i) If $F\left(\rho_{1}\right) \geq 0$ for some $\rho_{1} \in[0, \infty)$, then $\chi \rightarrow \infty, A \rightarrow \infty$ and $F \rightarrow \infty$ as $\rho \rightarrow \infty$.

(ii) If $F<0$ on $(0, \infty)$ then $F \rightarrow 0$ as $\rho \rightarrow \infty$. In addition, if $A$ and $Z$ have well-defined limits as $\rho \rightarrow \infty$ then $Z \rightarrow 0$ and $A \rightarrow A_{\infty}$ as $\rho \rightarrow \infty$, with $-1 \leq A_{\infty} \leq 1$

Similar results were established for the spherical case in [18]. Using these lemmas, boundary conditions were calculated under the assumption that the solutions were analytic in $1 / \rho$ at $\infty$. In [6] we showed that the numerical solutions in Figure 1 surround a centrally charged wire (in keeping with Lemma 1), and that the electron field is equal and oppositely charged, so that the total electric charge vanishes. (This last property was proven to be true of all stationary solutions in [19].)

\section{Vanishing total charge}

In the above solutions the variable $F(\rho)$ representing the total charge within a ring, radius $\rho$, tends towards zero at $\infty$. Lemma 1 also states that the potential must tend towards a solution which is logarithmic in $\rho$, near the central axis. This corresponds to a central charged wire (along the $z$ axis) which is a solution to the vacuum Maxwell equations. As such, we can think of this part of the solution as representing an external field. (The scalar potential could be separated at this point into $A_{\text {external }}+A_{\text {fermion+interaction, }}$ since the $A_{\text {external }}$ contributes nothing to the coupling between the Maxwell and Dirac equations.) Physically, this means that the (inner) external field must be surrounded by an equal and oppositely charged field. The total 

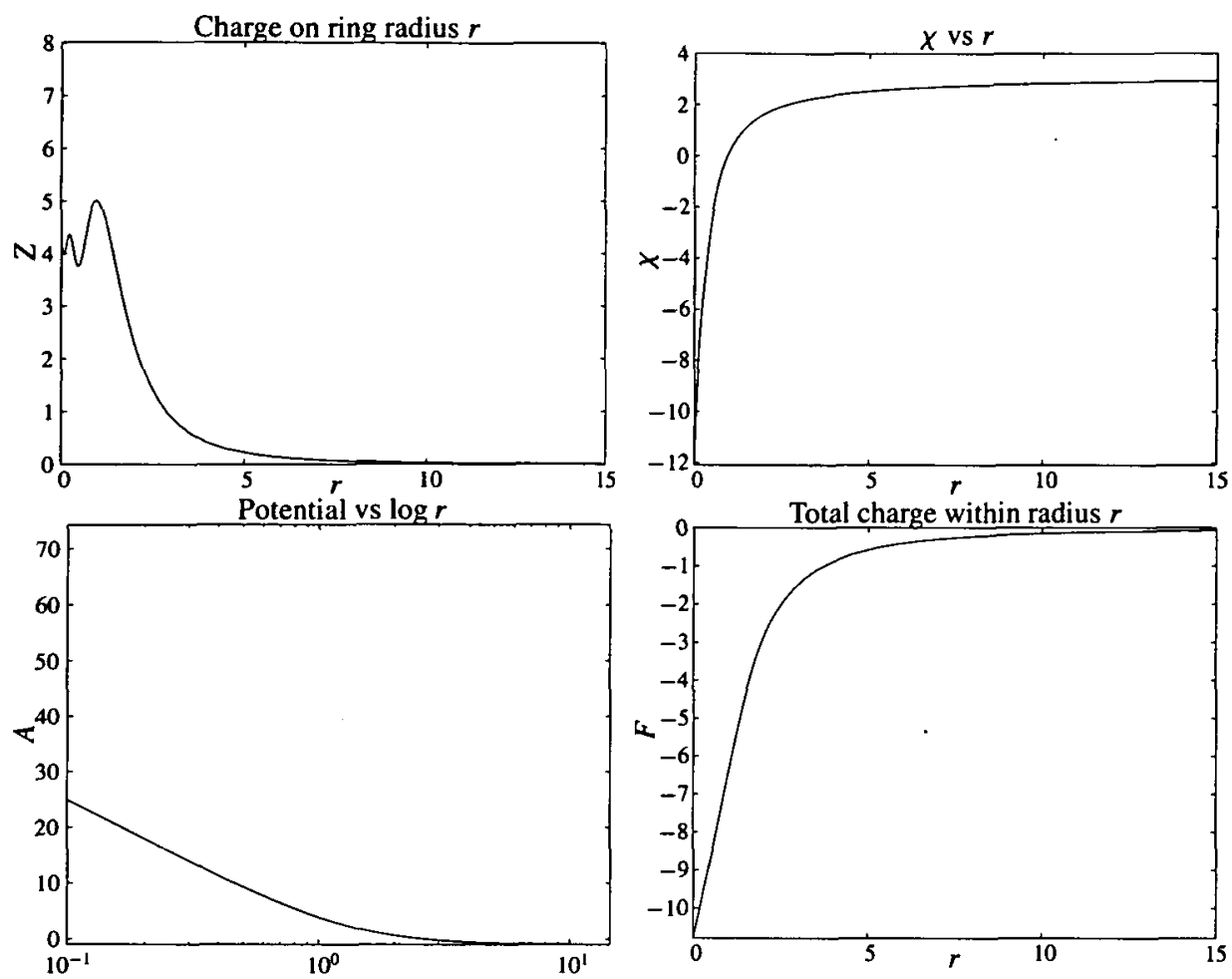

FIGURE 1. Localized solution to the cylindrically symmetric equations. The charge on a ring, radius $r, F(r)$, decreases to zero towards $\infty$. The potential (plotted against $\log r$ ) is $\log$-like at the origin, indicating a charged wire solution.

electric charge of the system

$$
\lim _{\rho \rightarrow \infty} \frac{1}{4 \pi} \int_{S_{\rho}}\left(\nabla A^{0}\right) \cdot d S, \text { with } S_{\rho} \text { the unit ring of radius } \rho
$$

must vanish.

In [3], we were able to show that this is the case for all stationary solutions, given that they are in a "reasonable" function space. If we define an isolated system as one for which all sources are contained in some ball $B_{k}(k<\infty)$ and for which the fields die off as $|x|=r \rightarrow \infty$, then we showed in [3] and [19] that an isolated, stationary, static Maxwell-Dirac system is electrically neutral. A similar result, for the total electric charge per unit length in the $z$ direction (or unit ring), holds in the cylindrical case. This shows mathematically that a (stationary) solution must be atom-like (in the sense that any central charge must be surrounded by an equal and opposite charge). In addition it shows that there cannot be a stationary solution representing an isolated electron (or even one that rotates around the axis with constant velocity). 

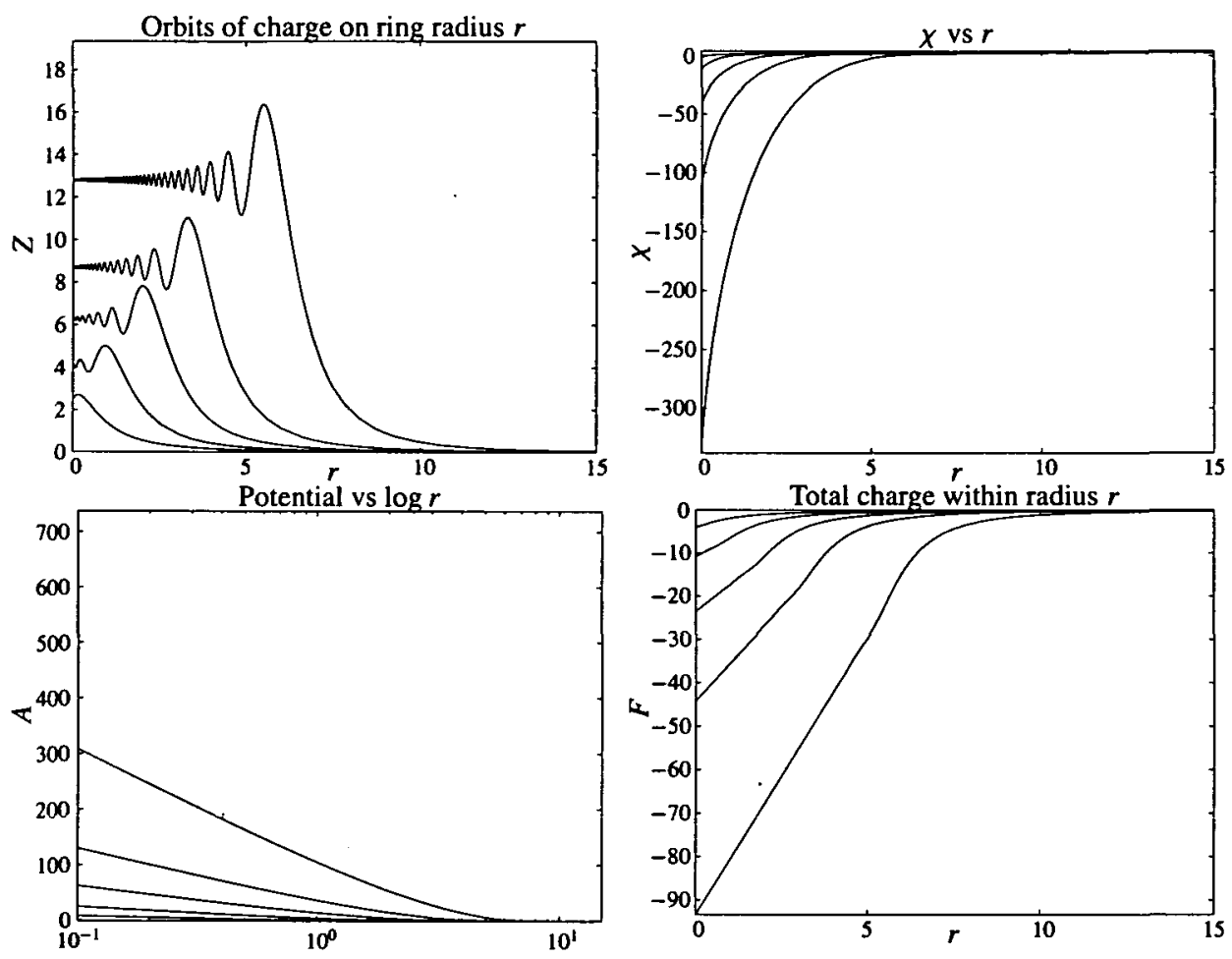

FIGURE 2. A family of orbits of which the solution in Figure 1 is an example. As nonlinear solutions they satisfy all the conditions of Lemmas 1 and 2 .

Note also that the signs of the charges can be reversed, giving us negatively charged singularities and positively charged "electron" fields. All results remain unchanged under such a reversal of sign. The total charge must vanish overall. A more difficult problem presents itself if we are to allow for two fermion fields of opposite charge, for example, the solution corresponding to positronium. We are currently considering ways in which we can have have solutions of opposite charge interacting together.

In [11] Esteban et al. showed that stationary solutions must be exponentially decreasing at infinity. We therefore looked for numerical solutions of this type. We assume that solutions are of the form $e^{-c|x|} g(|x|)$ where $g(|x|)$ is the analytic function which we solved for in the numerical examples above (in the cylindrical case). By varying the value of $c$, the boundary conditions are perturbed to neighboring solutions (orbits), all decreasing exponentially at infinity (see Figure 2).

The $c=0$ case is the numerical solution which we see in Figure 1. The other values of $c$ yield solutions which satisfy the two lemmas and the zero total charge result. That is, all solutions surround a central wire along the axis of symmetry. 


\section{Discussion and conclusions}

Similar extensions apply to the spherical case [4]. The spherical solutions surround a central Coulomb field, but the static condition forces a monopole at the origin $([3,18,19])$, that is, an unbounded $A^{\phi}$ component. Further ODE solutions occur in the $z$ case [3] and $1+1$ case, neither of which has been fully examined in the previous literature $([9,10])$. These results will be forthcoming also in [4].

Similar spherically-symmetric solutions were found in [17]. This time the Schrödinger-Newton equations provided an identical coupling as in the static Maxwell-Dirac case, which is essentially an elliptic system. More work must be done to investigate the stability of both of these systems. In [17] implications have been developed in the context of quantum gravity, but work in this area is far from complete. We are currently considering the problem in this context. The solutions found in [17] blow up at larger distances from the origin. However, in the Maxwell-Dirac case, this behaviour appeared only as a numerical anomaly. When the total charge became slightly positive (due to the step-size of the numerical solution), we entered a regime described in [6, Lemma 2] in which all solutions become unbounded as $\rho \rightarrow \infty$. These solutions were illustrated numerically in [6], but discarded as being of less interest than the bounded solutions.

The solution shown in Figure 1 was originally calculated using a NAG [23] routine converted into MATLAB $([6,22])$. The calculation was repeated using the MATLAB ODE solver ODE113. The numerical solutions shown in Figure 2 also made use of ODE113. The relative error tolerance was set at $1 e-4$ and the absolute error tolerance at $1 e-8$. The same behaviour was observed when the tolerances were decreased to $1 e-6$ and $1 e-12$ or increased to $1 e-3$ and $1 e-6$. All solutions remained stable whether calculated as a function of increasing or decreasing radius (that is, shooting away from or towards the central axis).

The two-dimensional cases (static axi-symmetric, circular current axi-symmetric, the massive $1+1$ case) still require reliable numerical results. Those available to date $([16,21])$ have been flawed by the imposition of "approximations" which were shown in [18] to be possible only in trivial cases (in which the equations are no longer coupled). The nonlinearity of this very interesting system forces localized solutions of specific types, and a case-by-case analysis is far from complete.

\section{References}

[1] M. J. Ablowitz, A. Ramani and H. Segur, "A connection between nonlinear evolution equations and ordinary differential equations of P-type. I", J. Math. Phys. 21 (1980) 715-721. 
[2] M. J. Ablowitz, A. Ramani and H. Segur, "A connection between nonlinear evolution equations and ordinary differential equations of P-type. II", J. Math. Phys. 21 (1980) 1006-1018.

[3] H. S. Booth, "The static Maxwell-Dirac equations", Ph. D. Thesis, University of New England, 1998.

[4] H. S. Booth, "Various ODE solutions to the static and 1+1 Maxwell-Dirac equations", (in preparation).

[5] H. S. Booth, P. Jarvis and G. Legg, "Algebraic solution for the vector potential in the Dirac equation", J. Phys. A: Math. Gen. 34 (2001) 5667-5677.

[6] H. S. Booth and C. J. Radford, "The Dirac-Maxwell equations with cylindrical symmetry", $J$. Math. Phys. 38 (1997) 1257-1268.

[7] J. Chadam, "Global solutions of the Cauchy problem for the (classical) coupled Maxwell-Dirac system in one space dimension", J. Funct. Anal. 13 (1973) 173-184.

[8] K. Cieliebak and E. Séré, "Pseudoholomorphic curves and multiplicity of homoclinic orbits", Duke Math. J. 77 (1995) 483-518.

[9] A. Das, "General solutions of Maxwell-Dirac equations in $1+1$ dimensional space-time and a spatially confined solution", J. Maths. Phys. 34 (1993) 3986-3999.

[10] A. Das and D. Kay, "A class of exact plane wave solutions of the Maxwell-Dirac equations", $J$. Maths. Phys. 30 (1989) 2280-2284.

[11] M. Esteban, V. Georgiev and E. Séré, "Stationary solutions of the Maxwell-Dirac and the KleinGordon-Dirac equations", Calc. Var. 4 (1996) 265-281.

[12] M. Flato, J. C. H. Simon and E. Taflin, Asymptotic Completeness, Global Existence and the Infrared Problem for the Maxwell-Dirac Equations, Memoirs of the American Mathematical Society (Amer. Math. Soc., 1997).

[13] V. Georgiev, "Small amplitude solutions of the Maxwell-Dirac equations", Indiana Univ. Math. J. 40 (1991) 845-883.

[14] R. T. Glassey and W. A. Strauss, "Conservation laws for the Maxwell-Dirac and Klein-GordonDirac equations", J. Math. Phys. 20 (1979).

[15] L. Gross, "The Cauchy problem for the coupled Maxwell and Dirac equations", Comm. Pure Appl. Math. 19 (1966) 1-5.

[16] A. G. Lisi, "A solitary wave solution of the Maxwell-Dirac equations", J. Phys. A 28 (1995) 5385-5392.

[17] I. M. Moroz, R. Penrose and P. Tod, "Spherically-symmetric solutions of the Schrödinger-Newton equations", Class. Quantum Grav. 15 (1998) 2733-2742.

[18] C. J. Radford, "Localised solutions of the Dirac-Maxwell equations", J. Math. Phys. 37 (1996) $4418-4433$.

[19] C. J. Radford and H. S. Booth, "Magnetic monopoles, electric neutrality and the static MaxwellDirac equations", J. Phys. A: Math. Gen. 32 (1999) 5807-5822.

[20] J. Schwinger, "Gauge invariance and mass. II", Phys. Rev. 128 (1962) 2425-2429.

[21] M. Wakano, "Intensely localized solutions of the Dirac-Maxwell field equations", Prog. T. Phys. 35 (1966) 1117-1141.

[22] MATLAB is a trademark of The Mathworks, Inc.

[23] NAG is a registered trademark of The Numerical Algorithms Group, Inc. 